\title{
DÜBLIN
}

Technological University Dublin

ARROW@TU Dublin

\section{Building a Risk Model for the Patient-centred Care of Multiple Chronic Diseases}

\author{
Stephane Deparis \\ IBM Research Ireland \\ Pierpaolo Tommasi \\ IBM Research Ireland \\ Alessandra Pascale \\ IBM Research Ireland
}

See next page for additional authors

Follow this and additional works at: https://arrow.tudublin.ie/engscheleart

Part of the Electrical and Computer Engineering Commons, and the Medicine and Health Sciences Commons

\section{Recommended Citation}

S. Deparis, P. Tommasi, A. Pascale, H. Rifai, J. Doyle and J. Dinsmore, "Building a Risk Model for the Patient-centred Care of Multiple Chronic Diseases," 2019 IEEE International Conference on Bioinformatics and Biomedicine (BIBM), 2019, pp. 1078-1082, doi: 10.1109/BIBM47256.2019.8983235.

This Conference Paper is brought to you for free and open access by the School of Electrical and Electronic Engineering at ARROW@TU Dublin. It has been accepted for inclusion in Conference papers by an authorized administrator of ARROW@TU Dublin. For more information, please contact arrow.admin@tudublin.ie, aisling.coyne@tudublin.ie, gerard.connolly@tudublin.ie.

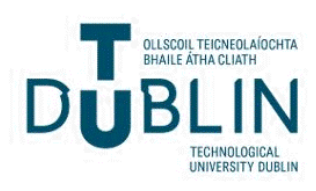


Authors

Stephane Deparis, Pierpaolo Tommasi, Alessandra Pascale, Hicham Rifai, Julie Doyle, and John Dinsmore 


\title{
Building a Risk Model for the Patient-centred Care of Multiple Chronic Diseases
}

\author{
Stephane Deparis*, Pierpaolo Tommasi*, Alessandra Pascale* ${ }^{*}$ Hicham Rifai ${ }^{\dagger}$, Julie Doyle ${ }^{\ddagger}$ and John Dinsmore ${ }^{\S}$ \\ *IBM Research - Ireland, Dublin, Ireland, stephane.deparis, ptommasi, apascale@ ie.ibm.com \\ ${ }^{\dagger}$ People Oriented Technology group, Technical University of Dublin, Dublin, Ireland, hicham.rifai@tudublin.ie \\ ${ }_{\ddagger}^{\ddagger}$ NetwellCASALA, Dundalk Institute of Technology, Dundalk, Ireland, julie.doyle@dkit.ie \\ $\S$ Trinity Centre for Practice in Healthcare Innovation, Trinity College Dublin, Dublin, Ireland, dinsmorj@tcd.ie
}

\begin{abstract}
With the increase of multimorbidity due to population ageing, managing multiple chronic health conditions is a rising challenge. Machine-learning can contribute to a better understanding of persons with multimorbidity (PwMs) and how to design an effective framework of care and support for them. We present a risk model of older PwMs that was derived from the TILDA dataset, a longitudinal study of the ageing Irish population. This model is based on a 26-nodes Bayesian network that represents patients possibly having one or more chronic conditions among diabetes, chronic obstructive pulmonary disease and arthritis, through a joint probability distribution of demographic, symptomatic and behavioral dimensions. We describe our method, give an exploratory analysis of the risk model, and assess its prediction accuracy in a crossvalidation experiment. Finally we discuss its use in supporting management of care for PwMs, drawing on comments from health practitioners on the model.
\end{abstract}

Index Terms-multimorbidity, Bayesian network, care management, risk model

\section{INTRODUCTION}

Advances in modern healthcare and medicine has lead to an increase in longevity, but living older is also positively correlated with managing multimorbidity, i.e., two or more chronic conditions [1]. A cross-sectional study of $1.8 \mathrm{M}$ Scottish patients found that $65 \%$ of patients older than 65 had two chronic conditions or more $(81.5 \%$ for patients older than 85) [1]. The most frequent conditions include: chronic obstructive pulmonary disease (COPD), diabetes, chronic heart failure (CHF), pain, depression and anxiety [2]. However, the medical literature stresses the lack of studies focused on multimorbidity. One key finding is that the more physical conditions a patient has, the more likely they are to suffer from mental health problems as well. In addition, multimorbidity has a multiplicative effect on the negative outcomes of the chronic diseases. Hence, addressing multimorbidity has potential in crucially increasing the quality-of-life for PwMs and decrease avoidable hospital admissions and readmissions, saving social and financial costs to society [3]. Finally, a review of randomized controlled trials involving

The research work is funded within ProACT by the H2020 program under grant agreement No. 689996. We thank all ProACT partners for productive discussions about this work.

978-1-7281-1867-3/19/\$31.00 @2019 IEEE multimorbidity showed that "interventions are likely to be more effective if targeted at risk factors or specific functional difficulties" [4].

Considering the importance of risk and the potential overlap of risk factors and symptoms in a situation of multimorbidity, we propose to build a risk model that encompasses several dimensions, from demographics and clinical to social and behavioural aspects. We aim at representing older persons with several chronic diseases, including diabetes, COPD, CHF and coronary heart disease (CHD). This model was developed in the context of ProACT, a European funded project set to design a home-based, digital integrated care system for persons with multimorbidity (PwMs in the following). The system is aimed at daily self-management of multimorbidity. The project gathers researchers in behavioural change, in risk analysis, in IoT as well as a network of informal carers, formal carers and healthcare professionals to carry trial studies on 120 patients from Ireland, Belgium and Italy. The PwMs are using wearable connected devices such as a blood-pressure and activity tracker watch and use a care application that records measurements, collects self-assessment reports and provides other functionalities such as an education recommender (see Doyle et al. for an overview of the project [5]). We present the risk model that was developed to support the project, and was learnt from the TILDA dataset, socio-economic and health data collected in a longitudinal study of the older Irish population. The risk model is a Bayesian network representing the joint probabilities of variables from the demographic, clinical and behavioural domain that were collected in TILDA. A preliminary risk model based on TILDA was presented in Deparis et al. 2018, that featured 10 variables [6]. The new risk model presented features 26 variables and we explore the clinical effects that can be seen in the model.

The paper is divided as follows: the next section presents our methodology. In the 2nd section, we present the developed risk model and review some of the observed dependences (e.g., "Arthritis (of any type) is a risk factor for abnormal heart rhythm") and how they concur with the clinical literature. In the 3rd section we assess the prediction accuracy of the model, comparing it to a naive uniform prediction, in a crossvalidation experiment using the Brier score. Finally, we discuss the use and relevance of the model in the last section. 


\section{Methodology}

\section{A. Dataset, data selection and processing}

Prior to the availability of trials data, we identified the TILDA (The Irish LongituDinal Study on Ageing) dataset, wave 1 , as representative of the trial population. It holds data for 8504 individuals, representative of the Irish population aged 50 and over, through a self-filled form, a computerassisted interview and a health assessment. It is available at http://www.ucd.ie/issda/data/tilda/. We further identified a subset of 26 variables that were related to multimorbid persons suffering from the target conditions: COPD, CHD, CHF, diabetes (see Table I). The time needed to run the learning algorithm (see subsection II-B) constrained the number of variables, and also lead us to choose for a categorical Bayesian network (BN in the following). When variables had continuous numeric values, they were discretized using categorical limits. These limits were defined using clinical guidelines (e.g. Blood pressure, $B M I$ ) or by binning the values to have groups with a sufficient size (e.g., Age, Years smoking). The variable Blood pressure was built out of diastolic BP and systolic BP, using logical disjunctions. Assets indicates to which quintile of the Gross Total Assets (GTA: sum value of main residence, savings, financial assets, etc.) distribution the person belongs to. Physical activity is measured through the IPAQ score, a standardized assessment of the level of physical activity [7]. Mental health problem refers to any clinically diagnosed mental condition, with anxiety and depression being the most largely represented conditions in the population. Social participation indicates if the person takes part in "any groups such as a sports or social group or club, a church connected group, a self-help or charitable body". Finally, Substance abuse indicates if a doctor ever diagnosed the participant with alcohol or substance abuse. When relevant, the number of missing values is different from the TILDA codebook because a legitimate skip of answer was considered as a False or No.

We assigned each variable to one of the following types:

Demographics describe basic information on the PwM like age, gender and socio-economic status.

Behaviours pertain to activities undertaken by the PwM, or describe aspects of their lifestyle, e.g. physical activity level or smoking habit.

Conditions are clinically diagnosed diseases that the PwM has to manage.

Vitals/Symptoms are vital readings, symptoms provided in self-reports, or related to conditions.

These types were used for adding constraints in order to speed the search for the structure of the model. They are also outlined by different colours in the user interface (UI) and graph for ease of reading, as iterations of the model were discussed frequently with ProACT partners, as well as clinical researchers (see V).

The mean age of the TILDA population is 63 years old, and out of the 8504 participants, a total of 2287 have arthritis and 3088 have hypertension. 641 have Diabetes, 337 have COPD, and only 89 persons have CHF. We observed that there is a frequent association between diabetes and hypertension. Finally, 725 participants have mental health problems.

We checked that the missingness for BMI and Blood pressure was not influenced by other variables of the model. To impute the missing data prior to learning, we ran experiments to compare k-nearest neighbours and random hot deck imputation methods. The former was the most accurate, especially when using $k$ close to the squareroot of the dataset size. However, it was also much slower, which lead us to use random hot-deck imputation for this work.

\section{B. Learning}

To learn a structure from the processed data, we applied the $A *$ algorithm. It is a scoring method based on the Minimum description length of the structure [8]. The TILDA dataset contains more than 1500 variables [9], however, the size of our risk model is constrained by the time needed to train it. For a structure search using only 22 variables, it takes on average 65 min 47 seconds to compute a directed acyclic graph (DAG) that represents the joint probability distribution of the data. To accelerate the search, we used a constraint graph [10] that restricts the search space. Using the types that we defined (see Table I), we enforced the following constraints: Demographics $\rightarrow$ all other types, Behaviours $\rightarrow$ Conditions and Vitals, and Conditions $\rightarrow$ Vitals.

\section{EXPLORING THE RISK MODEL}

Figure 1 represents the DAG resulting from learning using the constraints on domains. A Bayesian network structure is characterized by the Markov condition: any node is independent of its non-descendants, given its parents. Having that in mind, let us observe the learnt structure. All variables are connected, except for the small isolated subgraph formed by Assets and Social participation. Age and Gender are parents of a significant proportion of nodes, across the different types. We can easily distinguish some logical subgraphs which indicate the relevancy of specific conditions to associated symptoms or lifestyles. The subtree starting with Substance abuse, which appears linked to Mental health problem (essentially depression and anxiety in TILDA), themselves linked to chronic pain and problems in sleeping. The path going from Age to Receiving oxygen represents the increasing risk of requiring oxygen assistance with the duration of a smoking habit, through the increased risk of $C O P D$. Interestingly, the subgraphs related to a condition can intertwine, like the tree for Substance abuse, and the one for Arthritis. They indicate that a prediction for chronic pain will depend on the risk of arthritis, and/or mental health. Finally, we see that Hypertension and Diabetes are both parents of BMI and Number of daily medication.

In this subsection we give a non-exhaustive review of clinical effects observed in the TILDA constrained model, and how they corroborate or not clinical findings in the medical literature. We give the marginal effect of the parents going from the one state to the other (e.g. Gender observed being male to being female) while all other variables are kept in unobserved state. 
TABLE I: Variables in our Risk model. ADL stands for Activities of Daily Living, which include bathing, dressing, eating or climbing stairs.

\begin{tabular}{|c|c|c|c|c|c|}
\hline Index & Variable & Possible Values & \#missing & Type & $\begin{array}{c}\text { Brier Skill Score } \\
\text { (BN vs naive) }\end{array}$ \\
\hline 0 & Abnormal heart rhythm & False, True & - & Condition & 0.73 \\
\hline 1 & Age & below 60,60 to 70 , above 70 & $12(0.14 \%)$ & Demographics & 0.13 \\
\hline 2 & Arthritis & none, other, rheumatoid & - & Condition & 0.45 \\
\hline 3 & Assets & lvl1, lvl2, lvl3, lvl4, lv15 & $4381(51.52 \%)$ & Demographics & - \\
\hline 4 & Physical activity (IPAQ) & lvlo, lvl1, lvl2 & $81(0.95 \%)$ & Behaviour & 0.03 \\
\hline 5 & Blood pressure & normal, prehigh, high & $2393(28.14 \%)$ & Vitals/Symptoms & - \\
\hline 6 & Body Mass Index (BMI) & not obese, obese & $2377(27.95 \%)$ & Vitals/Symptoms & - \\
\hline 7 & $\mathrm{CHF}$ & False, True & - & Condition & 0.96 \\
\hline 8 & COPD & False, True & - & Condition & 0.87 \\
\hline 9 & Diabetes & False, True & - & Condition & 0.75 \\
\hline 10 & Falls & No, Yes & $2(0.02 \%)$ & Vitals/Symptoms & 0.36 \\
\hline 11 & Fear of falling & No, Yes & $3(0.04 \%)$ & Vitals/Symptoms & 0.37 \\
\hline 12 & Gender & $\mathrm{F}, \mathrm{M}$ & - & Demographics & 0.14 \\
\hline 13 & Hypertension & False, True & - & Condition & 0.26 \\
\hline 14 & Mental health problem & False, True & - & Condition & 0.68 \\
\hline 15 & $\mathrm{Nb}$ cigarettes per day & $0,1-9,10-19,20-39,40+$ & - & Behaviour & 0.13 \\
\hline 16 & Pain & False, True & $4(0.05 \%)$ & Vitals/Symptoms & 0.20 \\
\hline 17 & Pain medication & No, Yes & $1(0.01 \%)$ & Vitals/Symptoms & 0.48 \\
\hline 18 & Receiving Oxygen & False, True & - & Vitals/Symptoms & 0.98 \\
\hline 19 & Restless sleep & always, no, sometimes & $6(0.07 \%)$ & Vitals/Symptoms & 0.22 \\
\hline 20 & Smoker & currently, never, stopped & $1(0.01 \%)$ & Behaviour & 0.07 \\
\hline 21 & Years smoking & None, $1-9,10-19,20-29,30-39,40+$ & $51(0.60 \%)$ & Behaviour & 0.13 \\
\hline 22 & Social participation & No, Yes & - & Behaviour & 0.01 \\
\hline 23 & Substance abuse & False, True & - & Condition & 0.94 \\
\hline 24 & $\mathrm{Nb}$ of daily medications & $\operatorname{lv} 10$ to $\operatorname{lv} 13$ & - & Vitals/Symptoms & 0.13 \\
\hline 25 & $\mathrm{Nb}$ of $\mathrm{ADL}$ impairments & lv10 to $1 \mathrm{v} 15$ & - & Vitals/Symptoms & 0.82 \\
\hline
\end{tabular}

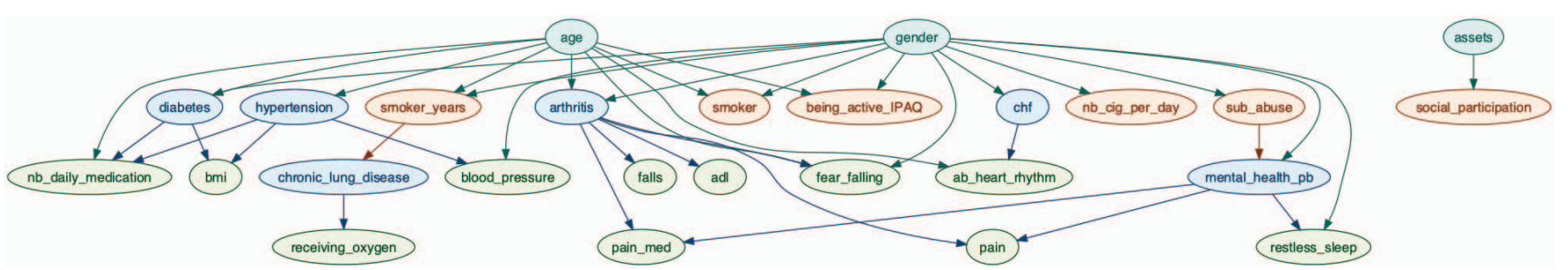

Fig. 1: DAG structure of the Bayesian Network learnt with constraints.

- Age increases Rheumatoid arthritis (below 60 vs above 70: $5.2 \%$ to $12.5 \%$ ). "The prevalence of RA was generally greater in the following groups: women, Mexican Americans, respondents with less education, and respondents who were 70 years of age and older." [11]

- Rheumatoid arthritis (RA) increases pain (25.8\% to $63.1 \%$ ). "Preferences for improved health examined in 1,024 patients with RA: Pain has highest priority." [12]

- Any arthritis increases abnormal heart rhythm (6.7\% to $8.1 \%$ ).

- Any arthritis increases ADL impairment, daily number of medication, fear of falling and falls. "we found increased odds of two or more falls for persons who had [...] arthritis" [13] "Fifty percent reported fear of falling, and $38 \%$ modified activities due to fear of falling." [14]

- Arthritis and Fear of falling decrease Physical activity "Independent risk factors for nonparticipation in physical activity were fear of falling (odds ratio $(\mathrm{OR})=0.70, p=$ .006)" [15] "Severity of arthritis is inversely associated with frequency of activity participation." [16]
- female gender increases the probability of mental health problems (male vs female: $6.6 \%$ to $10.1 \%$ ) "Women have consistently higher prevalence rates of anxiety disorders" [17]

- Substance abuse increases the probability of mental health problems $(8.1 \%$ to $35.6 \%)$. "Associations between most substance use disorders and independent mood and anxiety disorders were positive and significant." [18]

- Mental health problems increase restless sleep and pain "Significant positive associations were found between chronic pain and individual 12-month mood and anxiety disorders" [19]

A review of the clinical literature shows that the $\mathrm{BN}$ structure that was learnt represents many effects observed in clinical trials. Observing demographics information can significantly impact the probability estimates of conditions and symptoms. In addition, we observed that variables who have several conditions as parents in the DAG have very different risk distributions depending on the conjunction of states of the parents. For instance, the probability for complaints about pain 
varies from $24.4 \%$ to $62.4 \%$ to $69.9 \%$ when respectively none, rheumatoid arthritis, or a combination of rheumatoid arthritis and mental health problems are present. These effects indicate that the BN risk model is particularly relevant to assess risks for PwMs.

\section{ACCURACY OF THE RISK MODEL}

In the following, we evaluate the accuracy of the risk model. We run an experiment to observe, for many outcome variables, how well the $\mathrm{BN}$ predicts the real observed state of the variable. To evaluate accuracy, we use a cross validation on the TILDA dataset, using the Brier skill score as the measure of accuracy. The Brier score [20] is widely used to evaluate forecasts, in the meteorological domain for example. It is defined as the mean squared error between predictions and observations: $B r=\frac{1}{N} \sum_{n=1}^{N} \sum_{k=1}^{L}\left(p_{n k}-o_{n k}\right)^{2}$, where $N$ is the number of predicted instances and $L$ the number of levels the variable can take, $p_{n k}$ is the probability estimate of level $k$ for instance $n$, and $o_{n k}$ is the boolean outcome of level $k$ for instance $n . B r$ ranges from 0 , for a perfect prediction model to $L$, for a model that is always wrong. We compare our risk model to the baseline of a naive predictor who always assigns a uniform distribution for the predicted variable. To compare the $\mathrm{BN}$ model to the baseline, we computed the Brier skill score, relative to the naive baseline:

$$
B S S(B N \text { vs naive })=1-\frac{B r_{B N}}{B r_{\text {naive }}}
$$

where $B r_{B N}$ is the Brier score of the $\mathrm{BN}$ and $B r_{\text {naive }}$ is the Brier score of the naive method. The Brier skill score measures the improvement of the $\mathrm{BN}$ forecast, relative to the naive forecast. It ranges from $-\infty$ to 1 (perfect skill compared to the reference). Negative values indicate that the $\mathrm{BN}$ is less accurate than the naive method, and 0 would mean that there is no improvement.

Variables with more than $1 \%$ of missing values (BMI, Blood pressure, Assets) were excluded to keep the folds size consistent. Each value is the average of a 10 -fold cross validation, where $10 \%$ of the test fold is used. The results are presented in Table I, last column. The BN improves for all predictions over a naive guess (no negative score), but the improvements varies depending on the variable. It is highest for variables with less levels, and we observe a substantial improvement for many variables, including most Condition variables. The $\mathrm{BN}$ prediction accuracy is particularly high for $\mathrm{Nb}$ of $\mathrm{ADL}$ impairments, even though this variable has a high number of levels. These results indicate that the $\mathrm{BN}$ model is able to make good predictions even if it was learnt with minimal domain knowledge (the constraint graph) and with relatively little data given that it features 26 variables.

\section{DISCUSSION}

The work presented in this paper aims at building a $\mathrm{BN}$ risk model of PwMs. It represents the joint probability distribution of several variables describing a PwM, from demographic factors to the chronic conditions they may suffer from, and related symptoms or impairments. This model was learnt with minimal domain knowledge, using a scoring structure search algorithm on the TILDA data. Many probabilistic dependences represented corroborate the clinical literature. However, the high learning time lead us to limit the number of variables and to use categorical variables to describe a patient. Improvements could be gained by working more closely with healthcare professionals to use domain knowledge. In the following we discuss how the model can be used as part of a decisionsupport tool for care management of PwMs, how the model can be improved, and we present some feedback collected from healthcare professionals.

Possible uses of the risk model: The model was built prior to collecting trials data from the ProACT project. Future work will aim at learning a risk model based both on the TILDA data, and the trials data. A challenge lies in integrating these heterogeneous sources of data: the TILDA data is static and describes thousands of participants while the ProACT data is historical, with a huge proportion of missing data and covers 120 PwMS. The initial model that was built is encouraging in that it allows us to represent clinical effects, and the specific risks associated with a given combination of risk factors.

In the context of ProACT, the risk model is integrated as a backend that feeds other analytics: an education recommender and a physical activity goal recommender. The first one displays education tips based on the risk profile of the PwM. The second analytics suggests a weekly physical activity goal, in number of steps. Similar BN risk models representing PwM could be used to help for other tasks, e.g., identifying profiles of PwMs more prone to specific risks. Such a task would focus on observing what groups of risk factors are linked to a specific risk that requires attention from the care team, e.g. anxiety, or pain management.

In order to explore the model and discuss it with partners, we developed an interactive UI comprising a display of the graph as well as a table to explore all marginal probabilities. The marginals table is interactive: the user can select any state of any variable as observed, which updates all marginal probabilities in the table [6], [21].

The model represents probabilities for a given PwM, but the model output can also be interpreted as a description of a cohort of patients defined by the observations. For example, if the observations chosen are "gender: female", and "diabetes: true", the output of the model can be understood as a description of this specific subpopulation, where probabilities are a percentage of this population. A possible use of the model could be in risk stratification, and help in designing healthcare policies.

Feedback from health practitioners: The risk model and the explorer UI were presented during a workshop at the Trinity Biomedical Sciences Institute in Dublin, Ireland, in order to get feedback and discuss the clinical effects and the possible uses of the risk model. It was attended by 12 participants, mainly nurses and physiotherapists. We discuss some comments collected from the participants in the following.

The DAG shown on Figure 1 show that the variable Gross 
Total Assets is not a very useful information to predict other variables in the model. Other variables should be identified to appreciate the socio-economic status of patients, as recommended by the clinical professionals. They suggest to add more precise variables, e.g. income or health policy access. Income helps in understanding if the patient can provide for their self-care or if they need to receive assistance. Ethnicity, as it influences prevalence and symptoms for some conditions, and level of education were also suggested.

Nurses suggested to add characteristics of the accommodation as a valuable context, e.g., 'includes stairs or not' can help in assessing ease in mobility, physical activity, and fear of falling. The PwM living alone or not was considered a valuable variable as well, to understand if the patient's health and well-being are supported within their home, or at least by a close care network.

One of the main topics that emerged during the discussion on a support tool representing a PwM was the importance of contextualizing information. For instance, some participants stressed that "seeing numbers" or levels is not enough and that if a UI provides a patient profile, it would be "hugely helpful" if a baseline was provided, to make sense of each reading for a given patient. Providing context is an interesting challenge. Our models and associated UI already process data in order to provide meaningful categorical levels instead of numeric continuous measurements only (for example, "obese" instead of a specific value of the BMI). However, the participants agreed that even more context would be very valuable. Participants stated that "Context is queen" and that they prioritize questions like "What is normal for the patient?" and "Is last year better than this year?". Research aimed at representing PwMs should thus consider representing : a comparison of the PwM to a standard baseline (of a person that would be considered "normal") or a comparison between the current state of the PwM and a previous state considered as the status quo for this PwM. For example, in assessing the values for blood pressure or heart rate, the practitioners said they would want to know "what is high or low for the patient" in addition to the objective measurement and the self-report scores. These ideas indicate an interesting direction for future research.

\section{CONCLUSION}

A risk model representing persons with multimorbidity (PwMs) was built using the TILDA dataset, representative of the older Irish population. The risk model is a categorical Bayesian network containing 26 variables, that can take between two and six possible levels. They were chosen to represent PwMs across several dimensions including demographics, behaviours, symptoms, and the chronic conditions that the person has to manage, including diabetes and COPD. We presented the structure obtained running the $A *$ algorithm with a constraints graph on the types of variables to speed the search. We reviewed clinical effects that are represented in the model, and assessed its prediction accuracy relative to a naive predictor. Results show that the BN model is efficient for predicting the state of the variables but it can be improved by adding socio-economic variables. We discuss the use of the developed risk model as a back-end analytics for applications designed for technology-enabled patient self-care.

\section{REFERENCES}

[1] K. Barnett et al., "Epidemiology of multimorbidity and implications for health care, research, and medical education: a cross-sectional study," The Lancet, vol. 380, no. 9836, pp. 37-43, 2012.

[2] A. Prados-Torres, A. Calderón-Larranaga, J. Hancco-Saavedra, B. Poblador-Plou, and M. van den Akker, "Multimorbidity patterns: a systematic review," Journal of clinical epidemiology, vol. 67, no. 3, pp 254-266, 2014.

[3] J. L. Wolff, B. Starfield, and G. Anderson, "Prevalence, expenditures, and complications of multiple chronic conditions in the elderly," Archives of internal medicine, vol. 162, no. 20, pp. 2269-2276, 2002.

[4] S. M. Smith, H. Soubhi, M. Fortin, C. Hudon, and T. ODowd, "Managing patients with multimorbidity: systematic review of interventions in primary care and community settings," Bmj, vol. 345, p. e5205, 2012.

[5] J. Doyle et al., "Designing a proactive, person-centred, digital integrated care system," International Journal of Integrated Care, vol. 17, no. 5, 2017.

[6] S. Deparis, A. Pascale, P. Tommasi, and S. Kotoulas, "An analytical method for multimorbidity management using Bayesian networks," in Studies in Health Technology and Informatics, 2018, pp. 820-824.

[7] C. L. Craig et al., "International Physical Activity Questionnaire: 12country reliability and validity," Medicine \& science in sports \& exercise, vol. 35 , no. 8, pp. 1381-1395, 2003.

[8] C. Yuan, B. M. Malone, and X. Wu, "Learning optimal bayesian networks using A* search," in Proc. of the 22nd International Joint Conference on Artificial Intelligence (IJCAI), 2011, pp. 2186-2191.

[9] R. A. Kenny et al., "The design of the irish longitudinal study on ageing," Trinity College Dublin, Tech. Rep., 2010. [Online]. Available: http://tilda.tcd.ie/publications/reports/pdf/Report_DesignReport.pdf

[10] J. M. Schreiber and W. S. Noble, "Finding the optimal bayesian network given a constraint graph," PeerJ Computer Science, vol. 3, p. e122, 2017.

[11] E. K. Rasch, R. Hirsch, R. Paulose-Ram, and M. C. Hochberg, "Prevalence of rheumatoid arthritis in persons 60 years of age and older in the united states: effect of different methods of case classification," Arthritis \& Rheumatism: Official Journal of the American College of Rheumatology, vol. 48, no. 4, pp. 917-926, 2003.

[12] T. Heiberg and T. K. Kvien, "Preferences for improved health examined in 1,024 patients with rheumatoid arthritis: pain has highest priority," Arthritis Care \& Research, vol. 47, no. 4, pp. 391-397, 2002.

[13] M. C. Nevitt, S. R. Cummings, S. Kidd, and D. Black, "Risk factors for recurrent nonsyncopal falls: a prospective study," Jama, vol. 261, no. 18 , pp. 2663-2668, 1989.

[14] K. D. Fessel and M. C. Nevitt, "Correlates of fear of falling and activity limitation among persons with rheumatoid arthritis," Arthritis \& Rheumatism: Official Journal of the American College of Rheumatology, vol. 10, no. 4, pp. 222-228, 1997.

[15] D. G. Bruce, A. Devine, and R. L. Prince, "Recreational physical activity levels in healthy older women: the importance of fear of falling," Journal of the American Geriatrics Society, vol. 50, no. 1, pp. 84-89, 2002.

[16] Z. Zimmer, T. Hickey, and M. S. Searle, "Activity participation and wellbeing among older people with arthritis," The Gerontologist, vol. 35, no. 4, pp. 463-471, 1995.

[17] C. P. McLean, A. Asnaani, B. T. Litz, and S. G. Hofmann, "Gender differences in anxiety disorders: prevalence, course of illness, comorbidity and burden of illness," Journal of psychiatric research, vol. 45, no. 8, pp. 1027-1035, 2011.

[18] B. F. Grant et al., "Prevalence and co-occurrence of substance use disorders and independentmood and anxiety disorders: Results from the national epidemiologic survey on alcohol and relatedconditions," Archives of general psychiatry, vol. 61, no. 8, pp. 807-816, 2004.

[19] L. A. McWilliams, B. J. Cox, and M. W. Enns, "Mood and anxiety disorders associated with chronic pain: an examination in a nationally representative sample," Pain, vol. 106, no. 1-2, pp. 127-133, 2003.

[20] G. W. Brier, "Verification of forecasts expressed in terms of probability," Monthly Weather Review, vol. 78, p. 1, 1950.

[21] P. Tommasi, S. Deparis, and A. Pascale, "HWProfile UI: facilitating the exploration of a patient centred risk model," in 2019 IEEE International Conference on Healthcare Informatics (ICHI). IEEE, 2019, in press. 